\title{
Eigenvalue spectra of complex networks
}

\author{
G. J. Rodgers ${ }^{1}$, K. Austin ${ }^{1}$, B. Kahng ${ }^{2}$ and D. Kim ${ }^{2}$ \\ ${ }^{1}$ Department of Mathematical Sciences, Brunel University, \\ Uxbridge, Middlesex UB8 3PH, U.K. and \\ ${ }^{2}$ Center for Theoretical Physics and Department of Physics, \\ Seoul National University, Seoul, 151-742, Korea
}

\begin{abstract}
We examine the eigenvalue spectrum, $\rho(\mu)$, of the adjacency matrix of a random scale-free network with an average of $p$ edges per vertex using the replica method. We show how in the dense limit, when $p \rightarrow \infty$, one can obtain two relatively simple coupled equations whose solution yields $\rho(\mu)$ for an arbitrary complex network. For scale free graphs, with degree distribution exponent $\lambda$, we obtain an exact expression for the eigenvalue spectrum when $\lambda=3$ and show that $\rho(\mu) \sim 1 / \mu^{2 \lambda-1}$ for large $\mu$. In the limit $\lambda \rightarrow \infty$ we recover known results for the Erdös-Rényi random graph.

PACS numbers: 05.10.-a,05-40.a,05-50.+q,87.18.Sn
\end{abstract}

Keywords: Complex networks, replicas, eigenvalues 


\section{INTRODUCTION}

The properties of networks, and in particular random networks, underpin theories in a broad range of information systems, engineering, physical science and social science research areas. In the simplest random network, introduced by Erdös and Rényi [1], edges are introduced at random between a fixed number of vertices $N$. More recently, Watts and Strogatz [2] have introduced a small world network which contains both the local structure present in real space networks and the short average path length characteristic of random graphs. In addition, Barabási and Albert [3] have introduced a grown random graph, in which vertices and edges are added over time. This results in a graph that has a power law degree distribution, typical of the degree distribution measured in a range of real world networks [4].

In order to understand the properties of these scale free graphs, a number of authors have developed random graphs, that on the one hand can be treated by the methods of equilibrium statistical mechanics, but on the other have a power law degree distribution typical of a grown graph.

In particular Goh et al [5] have introduced a static model which defines a grand canonical ensemble for these graphs. Each vertex $i$ is assigned a weight $P_{i}$ and an edge is introduced between vertices $i$ and $j$ with probability $P_{i} P_{j}$. A parameter $p$, which plays the role of the chemical potential in the grand canonical ensemble, can be regarded as time when considering the correspondence between the static graph and a grown graph. As $p$ increases, so does the average number of edges per vertex. This model, which was studied in more detail in [6], is more amenable to study by analytical methods than conventional growing random networks.

There have been a number of studies of the eigenvalue spectrum of the adjacency matrix of scale free graphs. In [7] a numerical study of a Barabási-Albert [3] scale free graph found that the eigenvalue density of the adjacency matrix $\rho(\mu)$ decayed like $1 / \mu^{5}$ for large $\mu$. This study also found that in the centre of the spectrum the density was triangular, rather than semi-circular as in the Erdös-Rényi random graph.

In [8] a similar numerical study was undertaken, which obtained different results. In particular it was found that the eigenvalue spectrum decayed as $1 / \mu^{4}$ and that the behaviour in the centre of the spectrum was exponential. 
In [9] a family of random graphs was considered, which includes the static model introduced by Goh et al [5], and the conditions under which a giant component exists were derived.

Finally, anaytical work was undertaken in $[10,11]$. Here an exact expression was obtained for the eigenvalue density of a tree-like scale free graph with degree distribution exponent $\lambda$. When this was solved under a continuum approximation it was found that in the tail of the eigenvalue spectrum $\rho(\mu) \sim 1 / \mu^{2 \lambda-1}$.

In this paper we use the replica method to examine the eigenvalue spectrum of the adjacency matrix for an arbitrary complex network. We show how, in principle, the eigenvalue spectrum can be obtained for all complex networks, and recover known results for the Erdös-Rényi graph. In the high density, high average degree limit, we obtain a simpler set of equations which allow us to obtain the spectrum exactly for a complex network with a power law degree distribution with exponent 3. We also show how the tail of the eigenvalue spectrum is power-law with exponent $2 \lambda-1$ for a complex network with a power-law degree distribution with exponent $\lambda$.

In the next section we develop the analysis, and in Section III present our results for an arbitrary dense complex network and check that we recover known results for the ErdösRényi graph. In Section IV the results for a scale free graph are presented and in Section V we summarise our findings.

\section{SOLUTION BY REPLICAS}

We follow the prescription of the static graph introduced in Goh et al [5]. Suppose we have a fixed number of vertices $N$ and that each vertex $i=1, \ldots, N$ is given a probability $P_{i}$ with $\sum_{i} P_{i}=1$. The Erdös-Rényi graph corresponds to $P_{i}=1 / N$. To construct the graph we take $P_{i} \sim i^{-\alpha}$ so that the resulting graph has a power law degree distribution with exponent $\lambda=1+1 / \alpha$.

At each time step two vertices $i$ and $j$ are selected with probabilities $P_{i}$ and $P_{j}$. If vertices $i$ and $j$ are already connected, or $i=j$, then we do nothing. Otherwise, an edge is introduced between vertices $i$ and $j$. This process is repeated $p N / 2$ times, where $p / 2$ is the average number of edges per vertex. Hence the probability that vertex $i$ and $j$ are joined by an edge, 
$f_{i j}$, is

$$
f_{i j}=1-\left(1-2 P_{i} P_{j}\right)^{p N / 2} \sim 1-\exp \left(-p N P_{i} P_{j}\right) .
$$

When $N P_{i} P_{j}<<1$ for all $i \neq j$ which is the case when $0<\alpha<1 / 2$ or $\lambda>3, f_{i j} \sim 2 p N P_{i} P_{j}$. Then our $f_{i j}$ reduces to that studied e.g. in $[6,9]$.

The adjacency matrix $A$ of this network has elements $A_{i j}=A_{j i}$ with probability distribution

$$
P\left(A_{i j}\right)=f_{i j} \delta\left(A_{i j}-1\right)+\left(1-f_{i j}\right) \delta\left(A_{i j}\right) .
$$

Placing $P_{i}=1 / N$ gives the Erdös-Rényi random graph. In [12] the replica method was used to study the eigenvalue spectrum of a matrix which was equivalent to the adjacency matrix of the Erdös-Rényi graph, but where the non-zero elements took the values \pm 1 with equal probability. We will follow the method used in [12] and [13] by introducing a generating function

$$
Z(\mu)=\int_{-\infty}^{+\infty}\left(\prod_{i} d \phi_{i}\right) \exp \left(\frac{i}{2}\left(\mu \sum_{i} \phi_{i}^{2}-\sum_{i j} A_{i j} \phi_{i} \phi_{j}\right)\right)
$$

where in order to ensure convergence, $\mu$ has a small positive imaginary part. Then the average eigenvalue density $\rho(\mu)$ is given by

$$
\rho(\mu)=\frac{2}{N \pi} \operatorname{Im} \frac{\partial<\log Z(\mu)>}{\partial \mu}
$$

where $<$. $>$ denotes an average over the disorder in the matrix $A_{i j}$. To evaluate this average we use the replica trick and write the $n^{\text {th }}$ power of the average of $Z(\mu)$ as

$$
<Z^{n}(\mu)>=\int_{-\infty}^{+\infty}\left(\prod_{i, \alpha} d \phi_{i \alpha}\right) \exp \left\{\frac{i \mu}{2} \sum_{i, \alpha} \phi_{i \alpha}^{2}+\sum_{i<j} \log \left[1+f_{i j}\left(\exp \left(-i \sum_{\alpha} \phi_{i \alpha} \phi_{j \alpha}\right)-1\right)\right\},\right.
$$

where the replica index $\alpha=1, \ldots, n$. Following [14], this logarithm can be written in the form

$$
\sum_{i<j} \log \left[1+f_{i j}\left(\exp \left(-i \sum_{\alpha} \phi_{i \alpha} \phi_{j \alpha}\right)-1\right)\right]=p N \sum_{i<j} P_{i} P_{j}\left[\exp \left(-i \sum_{\alpha} \phi_{i \alpha} \phi_{j \alpha}\right)-1\right]+R .
$$

In [14], a general expression like the left hand side of Eq.(6) was considered and it was shown that the remainder $R$ can be neglected as, when $2<\lambda<3$ then $R<O\left(N^{3-\lambda} \log N\right)$, when $\lambda=3$ then $\left.R<O\left((\log N)^{2}\right)\right)$ and when $\lambda>3$ then $R<O(1)$.

As a result, the average of the $n^{t h}$ power of $Z(\mu)$ can be written as

$$
<Z^{n}(\mu)>=\int_{-\infty}^{+\infty}\left(\prod_{i, \alpha} d \phi_{i \alpha}\right) \exp \left(\frac{i \mu}{2} \sum_{i, \alpha} \phi_{i \alpha}^{2}+\frac{p N}{2} \sum_{i j} P_{i} P_{j} f\left(\sum_{\alpha} \phi_{i \alpha} \phi_{j \alpha}\right)\right) .
$$


with $f(x)=\exp (-i x)-1$. Following the method used in [12], we can expand $f\left(\sum_{\alpha} x_{\alpha}\right)$ as

$$
f\left(\sum_{\alpha} x_{\alpha}\right)=f(0)+\sum_{\alpha} \sum_{r} b_{r} x_{\alpha}^{r}+\sum_{\alpha<\beta} \sum_{r, s} b_{r s} x_{\alpha}^{r} x_{\beta}^{s}+\sum_{\alpha<\beta<\gamma} \sum_{r, s, t} b_{r s t} x_{\alpha}^{r} x_{\beta}^{s} x_{\gamma}^{t}+\ldots
$$

We introduce auxillary fields $q_{\alpha}^{(r)}, q_{\alpha \beta}^{(r, s)}, q_{\alpha \beta \gamma}^{(r, s, t)}, .$. , which are conjugate to $N \sum_{i} P_{i}\left(\phi_{i}^{\alpha}\right)^{r}$, $N \sum_{i} P_{i}\left(\phi_{i}^{\alpha}\right)^{r}\left(\phi_{i}^{\beta}\right)^{s}, N \sum_{i} P_{i}\left(\phi_{i}^{\alpha}\right)^{r}\left(\phi_{i}^{\beta}\right)^{s}\left(\phi_{i}^{\gamma}\right)^{t}, \ldots$ respectively, and then we can rewrite Eq.(7) as

$$
\begin{aligned}
<Z^{n}(\mu)>=\int & D \phi D q \exp \left(\frac{i \mu}{2} \sum_{i, \alpha} \phi_{i \alpha}^{2}-\frac{p N}{2}\left\{\sum_{\alpha} \sum_{r} b_{r}\left(q_{\alpha}^{(r)}\right)^{2}+\sum_{\alpha<\beta} \sum_{r, s} b_{r s}\left(q_{\alpha \beta}^{(r, s)}\right)^{2}+\ldots\right\}+\right. \\
& \left.+p N\left\{\sum_{\alpha} \sum_{r, i} b_{r} P_{i} q_{\alpha}^{(r)}\left(\phi_{i}^{\alpha}\right)^{r}+\sum_{\alpha<\beta} \sum_{r, s, i} b_{r s} P_{i} q_{\alpha \beta}^{(r, s)}\left(\phi_{i}^{\alpha}\right)^{r}\left(\phi_{i}^{\beta}\right)^{s}+\ldots .\right\}\right) .
\end{aligned}
$$

In the limit $N \rightarrow \infty$, the integrals over $D q$ can be evaluated to give

$$
\begin{gathered}
q_{\alpha}^{(r)}=\sum_{i} P_{i}\left[\left(\phi_{\alpha}\right)^{r}\right]_{\phi, i}, \\
q_{\alpha \beta}^{(r, s)}=\sum_{i} P_{i}\left[\left(\phi_{\alpha}\right)^{r}\left(\phi_{\beta}\right)^{s}\right]_{\phi, i},
\end{gathered}
$$

etc.. where $[.]_{\phi, i}$, which is dependent on the vertex index $i$, is defined by

$$
\left[A\left\{\phi_{\alpha}\right\}\right]_{\phi, i}=\frac{\int d^{n} \phi A\left\{\phi_{\alpha}\right\} \exp \left(\frac{i \mu}{2} \sum_{\alpha} \phi_{\alpha}^{2}+p N P_{i} g\left\{\phi_{\alpha}\right\}\right)}{\int d^{n} \phi \exp \left(\frac{i \mu}{2} \sum_{\alpha} \phi_{\alpha}^{2}+p N P_{i} g\left\{\phi_{\alpha}\right\}\right)}
$$

and

$$
g\left\{\phi_{\alpha}\right\}=\sum_{i} P_{i}\left[f\left(\sum_{\alpha} \phi_{\alpha} \psi_{\alpha}\right)\right]_{\psi, i}
$$

Notice that the saddle point values of $\left\{q_{\alpha}^{(r)}\right\},\left\{q_{\alpha \beta}^{(r, s)}\right\}$, etc.. have the same functional dependence on $P_{i}$ as the order parameters for the spin glass on a generalised complex network [14]. The generating function can now be written as

$$
\begin{aligned}
<Z^{n}(\mu)>=\int D & \exp \left(-\frac{p N}{2}\left\{\sum_{\alpha} \sum_{r} b_{r}\left(q_{\alpha}^{(r)}\right)^{2}+\sum_{\alpha<\beta} \sum_{r, s} b_{r s}\left(q_{\alpha \beta}^{(r, s)}\right)^{2}+\ldots\right\}+\right. \\
& \left.+\sum_{k} \log \int d^{n} \phi \exp \left\{\frac{i \mu}{2} \sum_{\alpha} \phi_{\alpha}^{2}+p N P_{k} g\left\{\phi_{\alpha}\right\}\right\}\right) .
\end{aligned}
$$

Consequently, we can obtain the eigenvalue density of this system from the equations

$$
g\left\{\phi_{\alpha}\right\}=\sum_{k} P_{k} \frac{\int d^{n} \psi\left(\exp \left(-i \sum_{\alpha} \psi_{\alpha} \phi_{\alpha}\right)-1\right) \exp \left(\frac{i \mu}{2} \sum_{\alpha} \psi_{\alpha}^{2}+p N P_{k} g\left\{\psi_{\alpha}\right\}\right)}{\int d^{n} \psi \exp \left(\frac{i \mu}{2} \sum_{\alpha} \psi_{\alpha}^{2}+p N P_{k} g\left\{\psi_{\alpha}\right\}\right)} .
$$


Using Eq.(4) and Eq.(14) it is simple to show that the average density of states is

$$
\rho(\mu)=\frac{1}{n \pi} \operatorname{Re} \frac{1}{N} \sum_{i=1}^{N} \sum_{\alpha}\left[\left(\phi_{\alpha}\right)^{2}\right]_{\phi, i} .
$$

Hence in principle we can obtain the average density of states for any static network by solving Eq.(15) for $g\left\{\phi_{\alpha}\right\}$ and using the result to obtain $\rho(\mu)$ from Eq.(16). Even using the fact that we expect these solutions to be replica symmetric, this is impossible in general. Instead we follow $[12,13]$ and look for solutions in the dense, $p \rightarrow \infty$ limit, when $g\left\{\phi_{\alpha}\right\}$ is both quadratic and replica symmetric. In particular, when $g\left\{\phi_{\alpha}\right\}$ takes the form

$$
g\left\{\phi_{\alpha}\right\}=\frac{1}{2} a(\mu) \sum_{\alpha} \phi_{\alpha}^{2}
$$

then we have a self-consistent solution to Eq.(15) when

$$
a=-\frac{1}{n} \sum_{k} P_{k} \sum_{\alpha}\left[\left(\phi_{\alpha}\right)^{2}\right]_{\phi, k} .
$$

In the limit $n \rightarrow 0$ Eq.(16) and Eq.(18) can both be simplified to yield

$$
\rho(\mu)=-\frac{1}{\pi} \operatorname{Re} \frac{1}{N} \sum_{k=1}^{N} \frac{1}{i \mu+p N P_{k} a}
$$

and

$$
a=\sum_{k=1}^{N} \frac{P_{k}}{i \mu+p N P_{k} a} .
$$

\section{RESULTS}

As in [12], in the limit $p \rightarrow \infty$ the leading term in the eigenvalue spectrum can be calculated by assuming that $g\left\{\phi_{\alpha}\right\}$ is quadratic and replica symmetric. In order to do this, we find it easier to re-parameterise Eq.(20) and Eq.(19) by introducing $b=i p a / \mu$ and $\mu^{2}=p E^{2}$. This gives

$$
b=\frac{1}{E^{2}} \sum_{k=1}^{N} \frac{P_{k}}{1-b N P_{k}}
$$

and

$$
\rho(\mu)=-\frac{1}{\mu \pi} \operatorname{Im} \frac{1}{N} \sum_{k=1}^{N} \frac{1}{1-b N P_{k}}=-\frac{1}{\mu \pi} \operatorname{Im} b \sum_{k=1}^{N} \frac{P_{k}}{1-b N P_{k}} .
$$

Using Eq.(21) this can be rewritten as

$$
\rho(\mu)=-\frac{E}{\pi \sqrt{p}} \operatorname{Im} b^{2} .
$$


Thus Eq.(21) and Eq.(23) give a simple set of exact equations for the eigenvalue spectrum of an arbitrary dense complex network. Placing $P_{k}=1 / N$ yields

$$
\rho(\mu)=\frac{1}{2 p \pi} \sqrt{4 p-\mu^{2}}
$$

the result obtained in [12] for the Erdös-Rényi graph in the limit $p \rightarrow \infty$. By considering a quartic term in Eq.(17) we can expand around this result to obtain the $O(1 / p)$ perturbative corrections to $\rho(\mu)$ in Eq.(24), in agreement with [12].

\section{SCALE FREE GRAPHS}

To calculate the eigenvalue spectrum of the static scale free graph, we must, in general, take

$$
P_{k}=(1-\alpha) N^{\alpha-1} k^{-\alpha}
$$

which gives a static graph with a power-law degree distribution with exponent $\lambda=1+1 / \alpha$ [5]. Inserting this form into Eq.(21) and replacing the summation by an integral in the limit $N \rightarrow \infty$ gives, after some rearrangement,

$$
b=\frac{\lambda-2}{E^{2}} \int_{0}^{1} \frac{u^{\lambda-2} d u}{u-(1-\alpha) b} .
$$

\section{A. $\alpha=1 / 2$}

When $\alpha=1 / 2, \lambda=3$, it is simple to evaluate the integral in Eq.(26) and show that $b$ satisfies

$$
b=\frac{1}{E^{2}}\left[1+\frac{b}{2} \log \left(1-\frac{2}{b}\right)\right] .
$$

Defining $b=2 /\left(1-r e^{i \theta}\right)$ gives an equation for $\theta$ as a function of $E$,

$$
W(\theta) \equiv \frac{\theta \cos \theta}{\sin \theta}-\log \left(\frac{\theta}{\sin \theta}\right)+2 E^{2}-1=0
$$

and $r=\theta / \sin \theta$. As $\theta \rightarrow 0, W(\theta) \rightarrow 2 E^{2}$ and as $\theta \rightarrow-\pi, W(\theta) \rightarrow-\infty$. Hence there is a non-trivial solution $\theta(E)$ of Eq. $(28)$ in the range $-\pi<\theta<0$ for all $E$. Thus the eigenvalue density of this network in the dense, $p \rightarrow \infty$, limit, is given by

$$
\rho(\mu)=-\frac{E}{\pi \sqrt{p}} \frac{8 \theta \sin ^{3} \theta(\sin \theta-\theta \cos \theta)}{\left(\sin ^{2} \theta-\theta \sin 2 \theta+\theta^{2}\right)^{2}}
$$


where $\theta(E)$ is given by Eq.(28). Note that Eq.(29) is correctly normalised so that

$$
\int_{-\infty}^{\infty} \rho(\mu) d \mu=1
$$

As $E \rightarrow 0$ then $\rho(\mu) \rightarrow 4 /(3 \pi \sqrt{p})$ and as $E \rightarrow \infty$ then $\rho(\mu) \sim 1 /\left(\sqrt{p} E^{5}\right)$. In Fig. 1 this eigenvalue density is plotted.

\section{B. General $\alpha$}

Although we cannot solve Eq.(26) explicitly for general $\alpha$, one can obtain the eigenvalue spectrum in the large $\mu$ limit. In this limit, the solutions for $b$ come in conjugate pairs and we should choose the one with the negative imaginary part. Putting $(1-\alpha) b=B^{\prime}-i B^{\prime \prime}$, where $B^{\prime}$ and $B^{\prime \prime}$ are both real, Eq.(26) becomes

$$
B^{\prime}-i B^{\prime \prime}=\frac{(1-\alpha)(\lambda-2)}{E^{2}} \int_{0}^{1} \frac{u^{\lambda-2}\left\{\left(u-B^{\prime}\right)-i B^{\prime \prime}\right\}}{\left(u-B^{\prime}\right)^{2}+\left(B^{\prime \prime}\right)^{2}} d u .
$$

When $E \rightarrow \infty, B^{\prime}$ and $B^{\prime \prime}$ are small. The leading order of the real part of the integral on the right hand side of Eq.(31) is obtained by setting $b=0$ while that of the imaginary part is obtained by using the fact that as $B^{\prime \prime} \rightarrow 0, B^{\prime \prime} /\left(\left(u-B^{\prime}\right)^{2}+\left(B^{\prime \prime}\right)^{2}\right) \sim \pi \delta\left(u-B^{\prime}\right)$. Thus the integral on the right hand side of Eq. $(31)$ is $1 /(\lambda-2)-i \pi\left(B^{\prime}\right)^{\lambda-2}$. Thus we have

$$
B^{\prime}=\frac{1-\alpha}{E^{2}} \text { and } B^{\prime \prime}=\pi(\lambda-2)\left(B^{\prime}\right)^{\lambda-1},
$$

to leading order. Using these relations and Eq.(23) we finally obtain

$$
\rho(\mu) \sim \frac{2}{\sqrt{p}} \frac{(\lambda-2)^{\lambda-1}}{(\lambda-1)^{\lambda-2}} \frac{1}{E^{2 \lambda-1}} \sim \frac{1}{\mu^{2 \lambda-1}}
$$

as $\mu \rightarrow \infty$. In the other limit, $\mu \rightarrow 0$, we can obtain a series by expanding Eq.(26) in $1 / b$. This yields

$$
\rho(\mu) \sim \frac{(\lambda-1)^{2}}{\pi \lambda(\lambda-2)}\left\{1-\frac{(\lambda-1)^{2}\left(\lambda^{4}+\lambda^{3}+9 \lambda^{2}-5 \lambda+10\right)}{8(\lambda-2)^{2} \lambda^{2}(\lambda+1)(\lambda+2)} E^{2}+\mathrm{O}\left(E^{4}\right)\right\},
$$

illustrating that the eigenvalue spectrum $\rho(\mu)$ is analytic in the center of the band.

\section{SUMMARY}

We have shown how the eigenvalue spectrum of the adjacency matrix of an arbitrary static graph can be obtained analytically using the replica method. This again reinforces 
the position of the replica method as a systematic approach to a wide range of contemporary questions within statistical physics. In particular we obtained a pair of simple exact equations which yield the eigenvalue spectrum in the high density, $p \rightarrow \infty$, limit for an arbitrary complex network. Using these equations, we obtained known results for the the Erdös-Rényi random graph and found the eigenvalue spectrum exactly for the $\lambda=3$ scale free network. This suggests that in the dense limit, the centre of the spectrum is convex, not triangular [7] or exponential [8], as suggested by numerical studies of finite density networks. In the tail of the distribution we found that $\rho(\mu) \sim 1 / \mu^{2 \lambda-1}$ in agreement with results obtained from a continuum approximation to a set of equations derived for a tree-like scale free graph $[10,11]$. This suggests that this form of the spectral tail may be universal for a range of scale free graphs, given that the same result was obtained analytically for both tree-like and dense graphs, which could be regarded as being at opposite ends of the "ensemble" of scale free graphs.

In [13] the spectral density of the Laplacian on an Erdös-Rényi graph was considered using the replica method. In the future, we intend to build on this work, and the techniques developed in this paper, to study the spectral density of the Laplacian on arbitrary complex networks. This will be an important development in the theory of transport and diffusion on complex networks.

\section{ACKNOWLEDGEMENTS}

We would like to thank the Royal Society, London, for financial support. This work is in part supported by the KRF Grant funded by the Korean government MOEHRD (R142002-059-010000-0). 
[1] P. Erdös and A. Rényi, Publ. Math. Inst. Hung. Acad. Sci. Ser. A. 517 (1960).

[2] D. J. Watts and S. H. Strogatz, Nature 393440 (1998).

[3] A.-L. Barabási and R. Albert, Science 286509 (1999).

[4] R. Albert and A.-L. Barabási, Rev. Mod. Phys. 7447 (2002).

[5] K.-I. Goh, B. Kahng and D. Kim, Phys. Rev. Lett. 87278701 (2001).

[6] D.-S. Lee, K.-I. Goh, B. Kahng and D. Kim, Nucl. Phys. B 696351 (2004).

[7] I. Farkas, I. Derényi, A.-L. Barabási and T. Vicsek, Phys. Rev. E 64026704 (2001).

[8] K.-I. Goh, B. Kahng and D. Kim, Phys. Rev. E 64051903 (2001).

[9] F. Chung and L. Lu, Ann. Combinatorics 6, 125 (2002).

[10] S. N. Dorogovtsev, A. V. Goltsev, J. F. F. Mendes and A. N. Samukin, Phys. Rev. E 68 046109 (2003).

[11] S. N. Dorogovtsev, A. V. Goltsev, J. F. F. Mendes and A. N. Samukin, Physica A 33876 (2004).

[12] G. J. Rodgers and A. J. Bray, Phys. Rev. B 373557 (1988).

[13] A. J. Bray and G. J. Rodgers, Phys. Rev. B 3811461 (1988).

[14] D.-H. Kim, G. J. Rodgers, B. Kahng and D. Kim, Phys. Rev. E 71056115 (2005). 


\section{Figure Caption}

Fig. 1. The eigenvalue spectrum, $\rho(\mu)$ against $E$, with $\mu=\sqrt{p} E$, for the scale free, $\lambda=3$, complex network in the dense, $p \rightarrow \infty$, limit. The inset is a double logarithmic plot of the eigenvalue spectrum in the tail, where the line has a slope of -5 . 